\title{
Alkaline Atoms Held with Optical Tweezers
}

\section{Three separate groups demonstrate the trapping of two-electron atoms in arrays of optical tweezers, opening up new opportunities for quantum simulation and many-body studies.}

\section{by Antoine Browaeys*}

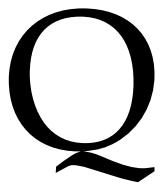

uantum technology is a very active field of research, in which the resources of quantum physics, such as superposition and entanglement, are utilized to perform tasks that are classically hard or impossible. The promises from this field are many, ranging from quantum computation of difficult mathematical problems to the building of more sensitive sensors. But to realize these possibilities, experimentalists have to engineer well-controlled quantum systems made of many individual elements that can interact with each other. Among the many platforms currently studied (ions, photons, electron spins, to name a few), neutral atoms have the advantage that they can be manipulated with high accuracy, and they are scalable in the sense that many thousands of atoms can be controlled. New experiments by three separate groups have

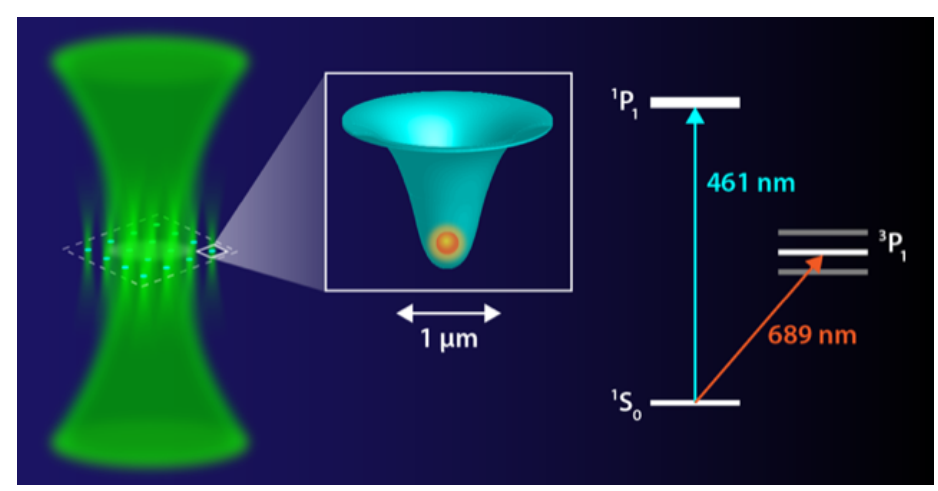

Figure 1: Two-dimensional arrays of optical tweezers can be created at the focal point of a laser beam (left). Each site in the array can trap a single atom (middle). For detecting and cooling, the atomic structure of strontium (right) features two types of transition: a strong one at $461 \mathrm{~nm}$ and a narrow one at $689 \mathrm{~nm}$. (APS/Alan Stonebraker) * Laboratory Charles Fabry, Institut d'Optique, CNRS, University
Paris-Saclay, Palaiseau, France raised the value of atom systems by demonstrating the trapping of alkaline-earth atoms inside an optical-tweezer array. Alkaline-earth atoms have two outer (or valence) electrons, giving researchers a rich atomic structure to exploit for quantum technology applications.

So far, most of the work with neutral atoms has been performed in optical lattices, in which the atoms are held at the nodes of an interference pattern between laser beams [1]. Starting from a gas of ultracold atoms, experimentalists can trap exactly one atom at each node, separated from its neighbor by half the wavelength of the laser $(\sim 500 \mathrm{~nm})$. But experimentalists are always looking for more flexibility and the ability to engineer more exotic situations.

Recently, an alternative approach has emerged, which relies on optical tweezers-a micrometer-sized optical trap obtained by focusing a laser beam with a high-numericalaperture lens. In some cases, the trapping volume is so small that, at most, one atom can be held in the trap [2]. Optical techniques can diffract the trapping beam into many spots, forming arrays of up to hundreds of atoms in a wide variety of two- and three-dimensional geometries (Fig. 1). Within the array, light beams can be used to shuffle atoms from one trap site to another [3]. In contrast to the fixed spacing in optical lattices, the typical distance between the atoms in an optical tweezer array can be varied from submicrometer to $\sim 10$ micrometers.

The interactions between atoms in an optical-tweezer array can be controlled by inducing quantum tunneling between array sites or by placing atoms in highly excited states, called Rydberg states. This interaction control has led to experiments exploring quantum magnetism $[4,5]$, or realizing quantum gates that are useful for quantum computations [6]. One nice feature of this tweezer approach relies on its fast duty cycle, where configurations can be reproduced at several $\mathrm{Hz}$, thus allowing for good statistics.

So far, tweezer-based platforms have operated only with alkali atoms, which possess one valence electron in their outermost shell. Working with alkali atoms poses some limitations. For example, it is quite hard to trap alkali Rydberg atoms in tweezers for a long enough time to manipulate them. An attractive alternative is found one column over in the periodic table with alkaline-earth atoms, such as stron- 
tium (Sr) and ytterbium $(\mathrm{Yb})$. Because these atoms have two valence electrons, they exhibit optical transitions with long lifetimes (from milliseconds to seconds) and wavelengths that span the optical spectrum from ultraviolet to infrared. Moreover, these transitions have broad linewidths that allow for fast cooling. Finally, the second valence electron makes it possible to trap the atom even when the first electron is in a Rydberg state.

Enticed by all these extra atomic "handles," three separate groups have succeeded in installing alkaline-earth atoms in optical-tweezer arrays. The first two teams-one led by Manuel Endres at the California Institute of Technology, Pasadena [7], and the other led by Adam Kaufman at JILA in Colorado [8] - were able to trap as many as several hundred strontium atoms inside optical-tweezer arrays. The third group from Jeff Thompson's lab at Princeton University, New Jersey, managed a similar feat with ytterbium atoms [9].

The trapping of alkaline-earth atoms in tweezers did not consist of simply tuning the lasers to different wavelengths. The whole "recipe" had to be rewritten. Indeed, the traditional method of loading alkali atoms into tweezers from a magneto-optical trap (MOT) does not work in the alkaline-earth case. For strontium, the two teams used a double-step loading procedure, which involved first precooling the atoms using a broad optical transition at $461 \mathrm{~nm}$ and then transferring the atoms into a so-called red MOT, operating on the narrow transition at $689 \mathrm{~nm}$. This standard practice, developed in the context of atomic clocks, resulted in a very cold and dense atomic sample. The two groups then overlapped the red MOT with an optical tweezer light pattern forming an array. This step required a careful management of the shifts induced by the tweezer light on the atomic states.

At the end of the loading procedure, each optical-tweezer site contained a few atoms, but only one atom per site is desired. To reach the single-atom regime, the teams applied a short pulse of light with wavelength of $461 \mathrm{~nm}$. This light induced the formation of short-lived two-atom molecules, which broke apart in such a way that the two liberated atoms were ejected from the trap. As a result, tweezer sites starting out with an even number of atoms were left empty, while sites with an odd number of occupants were whittled down to a single atom. As the initial atom number was randomly distributed between odd and even, there was a $50 \%$ chance of ending up with one atom at each tweezer site.

To detect which sites had a remaining atom, the researchers again had to be inventive. The common method would be to use fluorescence on the strong 461-nm transition, but that would result in rapid heating and loss of atoms. To counteract this heating, both of the strontium groups made 461-nm fluorescence measurements, while simultaneously performing a laser-cooling sequence with 689-nm light.
A similar set of steps was employed for the case of ytterbium. The only difference was that the ytterbium team used the same narrow transition at $556 \mathrm{~nm}$ for the cooling and the imaging. This is possible because the narrow transition in $\mathrm{Yb}$ is broader than for $\mathrm{Sr}$, which allows efficient cooling while scattering a lot of photons.

So far, only half the traps of the array are filled with individual atoms following the pulse-inducing light-assisted collisions, but the authors plan to try rearranging the atoms, using a recently demonstrated technique [3], to obtain a perfectly ordered atomic array. Complete arrays like this are ideal for performing quantum simulations. In previous investigations, researchers have proposed using alkalineearth-tweezer systems to explore many-body physics [10] and realize quantum-enhanced lattice clocks [11]. For now, the two-electron-atom trapping by the groups of Endres, Kaufman, and Thompson is an exciting development, exemplifying the good control experimentalists have achieved over neutral atoms. Many of us are expecting beautiful results with this new platform in the near future.

This research is published in Physical Review $X$ and posted on the arXiv.

\section{REFERENCES}

[1] C. Gross and I. Bloch, "Quantum simulations with ultracold atoms in optical lattices," Science 357, 995 (2017).

[2] N. Schlosser, G. Reymond, I. Protsenko, and P. Grangier, "Sub-poissonian loading of single atoms in a microscopic dipole trap," Nature 411, 1024 (2001).

[3] D. Barredo, S. de Léséleuc, V. Lienhard, T. Lahaye, and A. Browaeys, "An atom-by-atom assembler of defect-free arbitrary two-dimensional atomic arrays," Science 354, 1021 (2016); M. Endres, H. Bernien, A. Keesling, H. Levine, E. R. Anschuetz, A. Krajenbrink, C. Senko, V. Vuletic, M. Greiner, and M. D. Lukin, "Atom-by-atom assembly of defect-free onedimensional cold atom arrays," Science 354, 1024 (2016).

[4] H. Bernien et al., "Probing many-body dynamics on a 51-atom quantum simulator," Nature 551, 579 (2017).

[5] V. Lienhard, S. de Léséleuc, D. Barredo, T. Lahaye, A. Browaeys, Michael Schuler, L.-P. Henry, and A. M. Läuchli, "Observing the space- and time-dependent growth of correlations in dynamically tuned synthetic Ising models with antiferromagnetic interactions," Phys. Rev. X 8, 021070 (2018).

[6] D. S. Weiss and M. Saffman, "Quantum computing with neutral atoms," Phys. Today 70, No. 7, 44 (2017).

[7] A. Cooper, J. P. Covey, I. S. Madjarov, S.G. Porsev, M. S. Safronova, and M. Endres, "Alkaline-earth atoms in optical tweezers," Phys. Rev. X 8, 041055 (2018).

[8] M. A. Norcia, A. W. Young, and A. M. Kaufman, "Microscopic control and detection of ultracold strontium in optical-tweezer arrays," Phys. Rev. X 8, 041054 (2018).

[9] S. Saskin, J. T. Wilson, B. Grinkenmeyer, and J. D. Thomson, "Narrow-line cooling and imaging of ytterbium atoms in an optical tweezer array," arXiv:1810.10517. 
[10] R. Mukherjee, J. Millen, R. Nath, M. P. A. Jones, and T. Pohl, "Many-body physics with alkaline-earth Rydberg lattices," J. Phys. B 44, 184010 (2011).

[11] L. I. R. Gil, R. Mukherjee, E. M. Bridge, M. P. A. Jones, and T.
Pohl, "Spin squeezing in a Rydberg lattice clock," Phys. Rev. Lett. 112, 103601 (2014).

10.1103/Physics.11.135 